\title{
Learning Numbers in Different Languages: Chinese versus Malay
}

\author{
Natalie M. Y. Kheu \\ Terezinha Nunes \\ University of Oxford
}

This study investigated the impact of learning to use the Chinese or the Malay counting system on children's number development. Both languages are used as the medium of instruction in Malaysian national primary schools. It was predicted that Chinese learners would perform better than Malay learners on all number tasks because Chinese is a more regular counting system. Children $(N=120$; age 7 years) from two primary schools in Malaysia answered four number tasks (counting, hundred squares, a shop task, and a number line task) in their language of instruction. Chinese-speaking children outperformed their Malay-speaking peers in all tasks; the difference was statistically significant even after controlling for differences in socio-economic status and mother's education. The results supported the theory that the level of transparency of number systems influences children's ability to generate number names.

Keywords: Number systems, language, primary, Chinese, Malay

This study explores the structure of names for numbers in two languages: Malay and Chinese. It is hypothesized from past research that naming systems for numbers influences how a child learns and thinks about numbers. The learner's performance on number tasks varies depending on the regularity of the number system that one has to learn. A more transparent number system would logically be easier to acquire. The student would thus progress more quickly in their mastery of the number system.

The Malay and Chinese counting systems are more regular than English, but the Chinese system is the more regular of the two. As seen in Figure 1, the same word is used for 10 in Chinese, in the composition of numbers in the teens and in all other decades. In Malay, the word for 10, "sepuluh" (literally one ten) is not used in the composition of the teens; "puluh" is used in the composition of other decades, whereas in Chinese the same word is used. In Malay, the word for 1 is used with decades (e.g. 21, 31), but changed in the number labels for 10 and 11. The order in which the decades and units are named also differs between the systems: in Chinese both for the teens and for the other decades, the name of the unit is pronounced after the decade (as in twenty one) whereas in Malay 
the units are pronounced before the decade for numbers in the teens (as in eighteen, where the units come first).

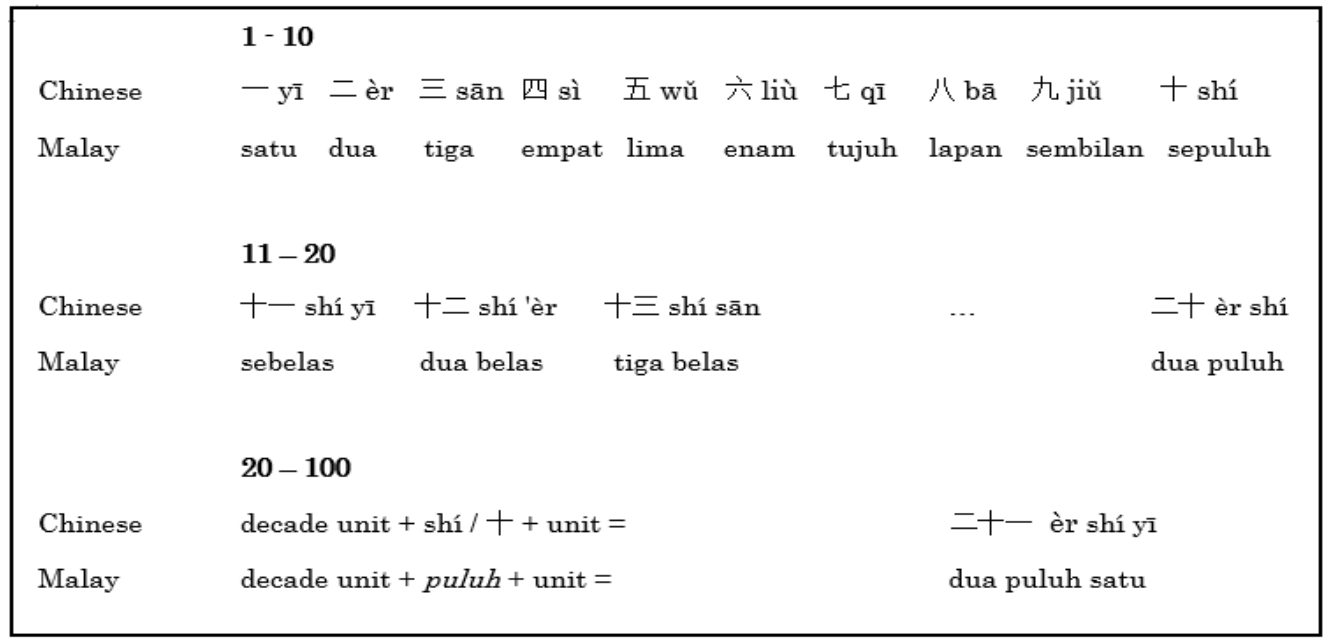

Figure 1. Rules for number formation in Chinese and Malay.

\section{Theoretical Framework}

This study's objectives were derived according to a simple but logical theoretical framework, as illustrated in the following two researches. The first investigation compared 69 Asian language speaking children (China, Japan, and Korea) and 70 non-Asian language speaking children (France, Sweden, and U.S) on how these 6 to 7 year old children cognitively represent numbers (Miura, Okamoto, Kim, Chang, Steere \& Fayol, 1994). The children were asked to construct numbers using blocks of different sizes (i.e., blocks representing ten units or one unit). The authors found that Asian language children would use base tens to construct numbers, and are able to construct the same number in two or more different ways (e.g., 4 tens and 2 units make 42, 3 tens and 12 units make 42). Non-Asian language children often used one-to-one collection (e.g., 42 units) and experienced difficulty conceiving two different ways to construct the same number. These differences were attributed to the numerical language characteristics which affected how the children cognitively represented numbers. What this indicates is that counting or number logic is the same in every culture (all children understand the logic of counting 42 units to make 42), but the organisation of the counting is different even if counting systems are constructed as base-10.

The second study by Seron and Fayol (1994) compared 8 to 9 years old French-speaking French and Belgian children on production of Arabic numbers. The children were asked to write down numbers that were orally dictated. The researchers found that the French children made more errors in comparison to the Belgian children. This was associated to the simplified Belgian number naming system, for example, Belgians use 'septante' for 
seventy whereas French use a more complicated name called 'soixante-dix' (sixty-ten) instead. The authors found that French-speaking Belgians completed additions quicker compared to their counterparts. What is clearly specified in this research is that counting systems, even with minor modifications in the same language, which are organised regularly are easier to learn. Thus, improving number representation in computation tasks. Despite originating from the same language, these differences in the number naming system show that the number system is a cultural artefact which has to be learned. This also provides a framework for the current study, predicting that the more complicated the number system, the more difficult it will be to apply number knowledge in number tasks.

In summary, children's cognitive representations of numbers are based on the naming system they use; therefore children who learn a more regular base10 counting system benefit as they understand how to construct numbers earlier on in their learning (Miura et al., 1994; Seron \& Fayol, 1994). Counting principles are learned around the same time by children of different cultures (Miller, Kelly, \& Zhou, 2005; Sui, 2012), but how far the children learn the counting string and how they structure their understanding of two digit numbers differs across cultures. Thus, it is hypothesized that children in a school learning mathematics with Chinese as a language of instruction (LOI) will learn the counting string further and understand its structure earlier on than those in schools with Malay as LOI.

\section{Number Concepts}

Understanding the structure of a counting system involves different aspects of numerical relations. The first is knowing the number naming system and its pattern and the second is additive composition, i.e. knowing that any number can be seen as the sum of two other numbers. A third aspect is understanding the place of a number in the counting system: for example, knowing that 54 is 10 more than 44 and between 53 and 56. This also relates to the distance between numbers: for example, knowing that 50 is halfway between 0 and 100 and that 90 is as close to 100 as 10 is to 0 .

\section{Number Naming Systems}

A study by Dowker, Bala, and Lloyd (2008) tested for linguistic differences in arithmetic ability. The experiment contrasted 60 Welsh children aged 6 and 8 years from a Welsh as LOI school (WW), an English as LOI school (EE), and another Welsh as LOI school with children who used English as their first language (WE) on their arithmetic abilities. The researchers administered several mathematical tests to them (e.g., British Ability Scales Basic Number Skills test) and a number-comparison task (i.e., read out pairs of numbers and say which is bigger) to test for calculation and arithmetic reasoning abilities. The results showed that there were no significant differences between the three 
schools in terms of global arithmetical ability, but there were differences in their ability to read and judge number pairs in the number-comparison task. In this, WW students outperformed WE students, and WE students in turn did better than EE students. This was explained by the regularity of the Welsh numerical system, for example, 11 in Welsh is un deg un ('one ten one'), 12 is un deg dau ('one ten two'), and 22 is dau ddeg dau ('two tens two'). This result is interesting as there is a similar cultural climate in Malaysia, in which there are schools using two different LOI, so any differences in the children's performance of number naming tasks could be related to the naming variances in the number system.

\section{Additive Composition}

Wang, Lin, Tanase and Sas (2008) did a study comparing the base-10 knowledge of 20 Chinese children (10 pre-schoolers and 10 first graders), 18 Romanian first graders, and 26 American first graders (aged 5 to 7 years). They used the methods utilised in Miura and colleagues' research by asking students to combine different types of blocks (e.g., base-10 blocks and unit blocks) to represent a number. The results revealed that Chinese children used base-10 blocks and outperformed both their Romanian and American counterparts, but the difference in performance between Romanian and American children were not significant. Both Romanian and American children preferred to use only unit blocks to construct numbers. Though Romanian is more transparent than English, the teens have several inconsistencies that could have influenced their base-10 knowledge (the number units change in the teens, and some numbers are flipped over/read back-to-front). This result could be extended to the present research in the Malay numerical system; the teens are also read back to front and a unit is interchanged with a shortened version for 11 (satu belas or sebelas), which makes this more difficult to learn.

Zhao, Valcke, Desoete, Burny, and Imbo's (2013) research compared 7247 Chinese and 913 Flemish students, aged 8 to 11 years old, on basic arithmetic performance (addition, subtraction, multiplication, and division) with a focus on linguistic differences. The authors predicted that if language differences were important, the difference in their performance would not become smaller over time. Counting in Flemish becomes slightly irregular during the teen numbers, and the numbers progress very similarly to English. For instance, 1 is één, but 11 is elf. 2 is twee, 12 is twaalf. From 13 onwards, tien is added behind the numbers 3 to 9 with modifications (e.g., 3 is drie and 10 is tien 13 is dertien). As Flemish counting is similar to English in terms of numerical scheme transparency, Chinese students were predicted to outperform Flemish students. Notably, even after matching participants for achievement motivation, they found that Chinese outperformed Flemish children in all tasks. This pointed strongly towards language for influencing the differences, which is what this study explores in terms of Chinese and Malay languages. 


\section{Number Placement}

For the number concepts of placement and distance, the number line is a useful tool designed to measure numerical estimation and is typically a straight line marked 1 and 100 at the ends (Siegler \& Booth, 2004). In a study by Siegler and $\mathrm{Mu}$ (2008), they examined whether Chinese kindergarteners' number line estimates would be more accurate and more linear than American children. They reasoned that Chinese children have superior counting skills to their American peers, so are likely to perform well on number estimations. The researchers included 29 Chinese children and 24 American children (aged 5 to 6 years) who were recruited from the respective countries, to compare their number estimation accuracy on a number line. They asked children to estimate 26 numbers on a 1 to 100 line, and also gave the children tasks involving addition. The researchers predicted that Chinese children would perform better than their American counterparts in both tasks, and the results they found supported this. The Chinese children's estimates were best represented by a more linear curve, while the American children had a more logarithmic curve. Once again there appears to be an advantage to using the Chinese language number system, as children seem to understand the size of estimates better. A potential biasing factor in this study is that the children were from two different countries, so there are various other influences like type of schooling that could have affected the children's performance, as cultural differences may affect representations of numbers in the brain (Bender \& Beller, 2011). The present study reduced this bias as the research evaluated the Chinese and Malay language in a similar cultural context.

In a more recent study, the roles of language and education in relation to number line estimation and simple arithmetic skills (addition) were investigated on two groups of children: Chinese groups which consisted of 41 kindergarteners and 31 second graders, and Chinese American groups made up of 42 kindergarteners and 20 second graders around the ages of 5 to 8 years (Laski \& Yu, 2014). The Chinese children were tested in their native language, while the Chinese Americans were tested in English (though noted to be fluent in both languages). Expectedly, the researchers found that Chinese kindergarteners performed better on number line estimation compared to Chinese American kindergarteners, but there was no difference between both groups of second graders' estimates. They mentioned that Chinese American second grader's estimates were also more accurate compared to Siegler and Booth's (2004) second grader's estimates, which offers interesting suggestions for language influence in bilinguals. This information is pertinent to the current study as the children in Malaysia are usually bilingual or multilingual. Laski and $\mathrm{Yu}$ also note from their results that a transparent base-10 counting system may assist in an earlier understanding of the magnitude of large numbers that persists with age even across dissimilar educational contexts. This implication is interesting especially if this result was found in Malay and Chinese performances, as both systems use base-10 but have differing levels of 
transparency. The authors also mentioned that there were curricular and instructional differences which had a significant influence on numerical knowledge. This is a factor that the current study will take into account by enlisting schools that use the same curriculum.

\section{Research Questions and Predictions}

There is a trend in research showing that the Chinese number naming system provides an advantage over many other language systems. A clear inclination in previous research is that Chinese children would perform better in numerical tasks because of their straightforward numerical system, and that number systems are cultural artefacts that have to be learned. In Malaysia, Chinese and Malay are both languages used as mediums of instructions in national schools, so it would be appropriate to explore further the relation of these languages to children's number knowledge.

The comparison makes a significant contribution to the literature for two reasons. First, all the children live in the same country, in multilingual environments, and attend schools that have the same curriculum, but delivered in different languages. Thus the samples are more comparable than those in previous studies, in which the groups under comparison lived in radically different cultures (Miller et al, 2005; Siegler \& Mu, 2008; Zhao et al., 2013). Second, the difference between the counting systems is much smaller than those in previous studies; thus the results can show whether even if a small difference in the level of regularity between the counting systems has a noticeable effect on children's learning.

The research questions for this study are:

(a) Would the language of instruction (Malay or Chinese) produce a difference in number task performance?

(b) Would the LOI produce a difference in making certain types of counting and shop task errors? According to the theoretical perspectives and literature review, the following predictions were derived:

(a) Chinese LOI participants will perform significantly better on all number tasks in comparison to Malay LOI participants.

(b) Chinese LOI participants will commit significantly less counting type errors and shop task errors in comparison to Malay LOI participants.

\section{Methods}

The study used a quasi-experimental design, in which the groups were not randomly assigned, but self-assigned (Bryman, 2012). Data were collected through purposive sampling, in which the schools approached would be representative of each LOI, Malay or Chinese. LOI is the main categorical and independent variable of the study. The children's scores in the different measures are the dependent variables. Mother's education was controlled for as 
previous research has shown that this has an effect on children's numerical knowledge at school entry (Dickson \& Robinson, 2013).

\section{Participants}

Participants $(N=120)$ were recruited from two state-supported schools in a suburban area in Malaysia, 60 from a school with Malay as LOI and 60 from a school with Chinese as LOI in mathematics lessons. All participants were in Grade 1 or the first formal year in primary school (aged 6 to 7 years; 62 boys and 58 girls). Other variables included in the analysis were parents' education, parents' occupation, and consistency in language use between school and the home.

\section{Measures}

A code-switching task was also used to check whether the children used the LOI in a simulation of everyday interactions. For instance, with a picture, the student role-played as a shopkeeper and the researcher as a customer asking for the price of items in their respective languages. Code-switching was noted if the language used by the child to reply was different than the LOI.

Knowledge of the counting string was measured by asking children to count objects in a row (10 each row) up to 70, counting on from 100 and 1000, and counting backwards from 20 (Miller \& Stigler, 1987) (see Appendix A). Types of counting errors like skip errors were also recorded.

Knowledge of the place of a number in the counting system was assessed by asking children to say the missing numbers in a hundreds board, which has been used in the literature to assess and improve children's understanding of numbers (Cobb, Wood, Yackel, \& McNeal, 1992). There were thirteen numbers missing from the box and the direction of reading was also noted (see Appendix B).

Additive composition was assessed by the Shop Task (Nunes \& Bryant, 1996), in which children are asked to compose specified amounts of money using coins of different values (combining 1,5 , and 10 money values to pay exact price for items) (see Appendix C). For example, paying an amount of 13 with money notes set of three $5 \mathrm{~s}$ and ten $1 \mathrm{~s}$. Types of money counting errors were recorded as well.

Knowledge of the distance and order of numbers was assessed using estimates of the position of numbers on a numbers line from 0 to 100 (Laski \& Siegler, 2007). This consists of asking the children to mark on a $26 \mathrm{~cm}$ line the place of a number on a line marked with 0 and 100 (Siegler $\&$ Mu, 2008). There were twenty-six randomly ordered numbers to place (see Appendix D).

These tasks aimed to address the research question of whether LOI would produce a difference in children's performance on various number tasks. The counting string and additive composition tasks were also utilised to discover whether making certain types of errors were related to differences in LOI. 


\section{Data Analysis}

The aim is to find if there are differences in LOI (independent variable) in terms of task outcomes (dependent variable). The method used to analyze differences between LOI for counting tasks is Pearson's chi-square; all the tasks met the assumptions of a chi-square test as they had an expected frequency of more than 5 participants per cell (Field, 2013; Pallant, 2013). The variables or the scores for the hundred squares task, shop tasks and number line tasks were found to be non-normally distributed. As the variables did not fulfil normality assumptions, a non-parametric test called the Mann-Whitney U-test was utilised.

\section{Results}

There were initial differences in the distribution of mother's education between the groups, which have been noted to impact children's numerical abilities (Dickson \& Robinson, 2013); a matching process was used to make the samples comparable, which reduced the sample to 86 participants. All subsequent analyses were based on this sample.

\section{Counting}

A chi-square analysis revealed that Chinese LOI children performed better significantly in counting to 70 and counting from $1000\left(X^{2} 1,86=12.77, p<\right.$ $.001)$; the direction of the difference was the same in the other tasks but not significant (see Table 1). Chinese LOI children made fewer counting errors than Malay LOI children too. Using chi-square analysis, it was found that Malay LOI children were more likely to commit skip errors when counting $\left(X^{2}{ }_{1,86}=\right.$ $11.06, p<.001$ ), for example, counting 10, 12, 13.

Table 1

Crosstabulation of LOI and Counting Tasks $(n=86)$

\begin{tabular}{|c|c|c|c|}
\hline \multirow{2}{*}{ Tasks } & \multicolumn{2}{|c|}{ Language of Instruction } & \multirow{2}{*}{$X^{2}$} \\
\hline & Malay & Chinese & \\
\hline \multicolumn{4}{|l|}{ Count to 70} \\
\hline Correct & 26 & 40 & \multirow{2}{*}{$12.77 * * *$} \\
\hline Incorrect & 17 & 3 & \\
\hline \multicolumn{4}{|c|}{ Count from 100} \\
\hline Correct & 5 & 11 & \multirow{2}{*}{2.76} \\
\hline Incorrect & 38 & 32 & \\
\hline \multicolumn{4}{|c|}{ Count from 1000} \\
\hline Correct & 4 & 11 & \multirow{2}{*}{$3.96^{*}$} \\
\hline Incorrect & 39 & 32 & \\
\hline \multicolumn{4}{|c|}{ Backwards from 20} \\
\hline Correct & 24 & 27 & \multirow{2}{*}{0.43} \\
\hline Incorrect & 19 & 16 & \\
\hline
\end{tabular}

Note. ${ }^{* * *}=p<.001,{ }^{*}=p<.05$ 


\section{Hundred Squares}

A comparison using the Mann-Whitney U-test of the total of correct answers between the groups revealed that they differed significantly ( $U=656$, $p<.01$ ) (see Table 2). Chinese LOI children were more likely to perform correctly on the hundred squares task regardless of which direction of reading they chose (by column $(11,21)$ or row $(10,11))$. This suggests that they understood the logic of the base-10 number system.

Table 2

Mean Ranks of Hundred Squares Task Performance by LOI (n = 86)

\begin{tabular}{|c|c|c|c|c|}
\hline & \multicolumn{2}{|c|}{ Language of Instruction } & \multirow[t]{2}{*}{$U$} & \multirow[t]{2}{*}{$r$} \\
\hline & Malay & Chinese & & \\
\hline Hundred & 37.26 & 49.74 & $656 * *$ & -0.33 \\
\hline Squares & $(13)$ & $(13)$ & & \\
\hline
\end{tabular}

Note. ${ }^{*} *=p<.01$, median in brackets.

\section{Additive Composition Shop Task}

The combined score of the shop task items consisting of fives and ones and tens and ones were summed for a total score (as children who passed fives and ones also passed tens and ones). This score was used in the comparison between the two groups utilising the Mann-Whitney U-test; the difference between the groups was significant $(U=520, p<.001)$ (see Table 3$)$. Chinese LOI children obtained higher scores when required to combine fives and ones as well as tens and ones. Chinese LOI children would pay 2 fives and 8 ones or 3 fives and 3 ones, but the majority of Malay LOI children paid 2 fives and 8 ones or miscounted fives as ones. Chi-square analysis found that Chinese LOI children were less likely to make errors of counting fives as ones (e.g., giving 5 fives and 8 ones to make 13) and tens as ones than Malay LOI children $\left(X^{2}{ }_{1,86}=8.32\right.$, $p<.01)$.

Table 3

Mean Ranks of Shop Task Performance by LOI $(n=86)$

\begin{tabular}{|c|c|c|c|c|}
\hline & \multicolumn{2}{|c|}{ Language of Instruction } & \multirow[t]{2}{*}{$U$} & \multirow[t]{2}{*}{$r$} \\
\hline & Malay & Chinese & & \\
\hline Sum of Shop & 34.09 & 52.91 & $520 * * *$ & -0.39 \\
\hline Task & $(3)$ & (5) & & \\
\hline
\end{tabular}

Note. $* * *=p<.001$, median in brackets.

\section{Number Estimation on Number Line}

In the number line task, two comparisons of the children's performance were carried out. In the first, the absolute error in the placement of the estimate was used. A Mann-Whitney U-test showed that Chinese LOI children produced lower absolute errors than Malay LOI children $(U=405, p<.001)$ (see Table 
4). This means that Chinese LOI children estimated numbers more accurately than their counterparts.

The second comparison was of the shape of the estimates, which show a linear increase with the value of the number to be estimated when children understand the number system (Siegler \& Booth, 2004). Both the Malay and Chinese LOI children's estimate lines were found to have a significant fit with a linear trend (see Figure 2). Both groups also showed a drop in accuracy when numbers in the teens were estimated, but the drop was more noticeable for the Malay LOI children. Both groups tended to underestimate the higher numbers, however comparatively, the Chinese LOI group placed the larger estimates more accurately on the line.

Table 4

Mean Ranks of Number Line Absolute Error of Estimates by LOI

\begin{tabular}{|c|c|c|c|c|}
\hline \multirow[t]{2}{*}{$(n=86)$} & \multicolumn{2}{|c|}{ Language of Instruction } & \multirow[t]{2}{*}{$U$} & \multirow[t]{2}{*}{$r$} \\
\hline & Malay & Chinese & & \\
\hline $\begin{array}{l}\text { NL Absolute } \\
\text { Error }\end{array}$ & $\begin{array}{l}55.58 \\
(5.06)\end{array}$ & $\begin{array}{l}31.42 \\
(2.90)\end{array}$ & $405 * * *$ & -0.48 \\
\hline
\end{tabular}

Note. $* * *=p<.001$, median in brackets.

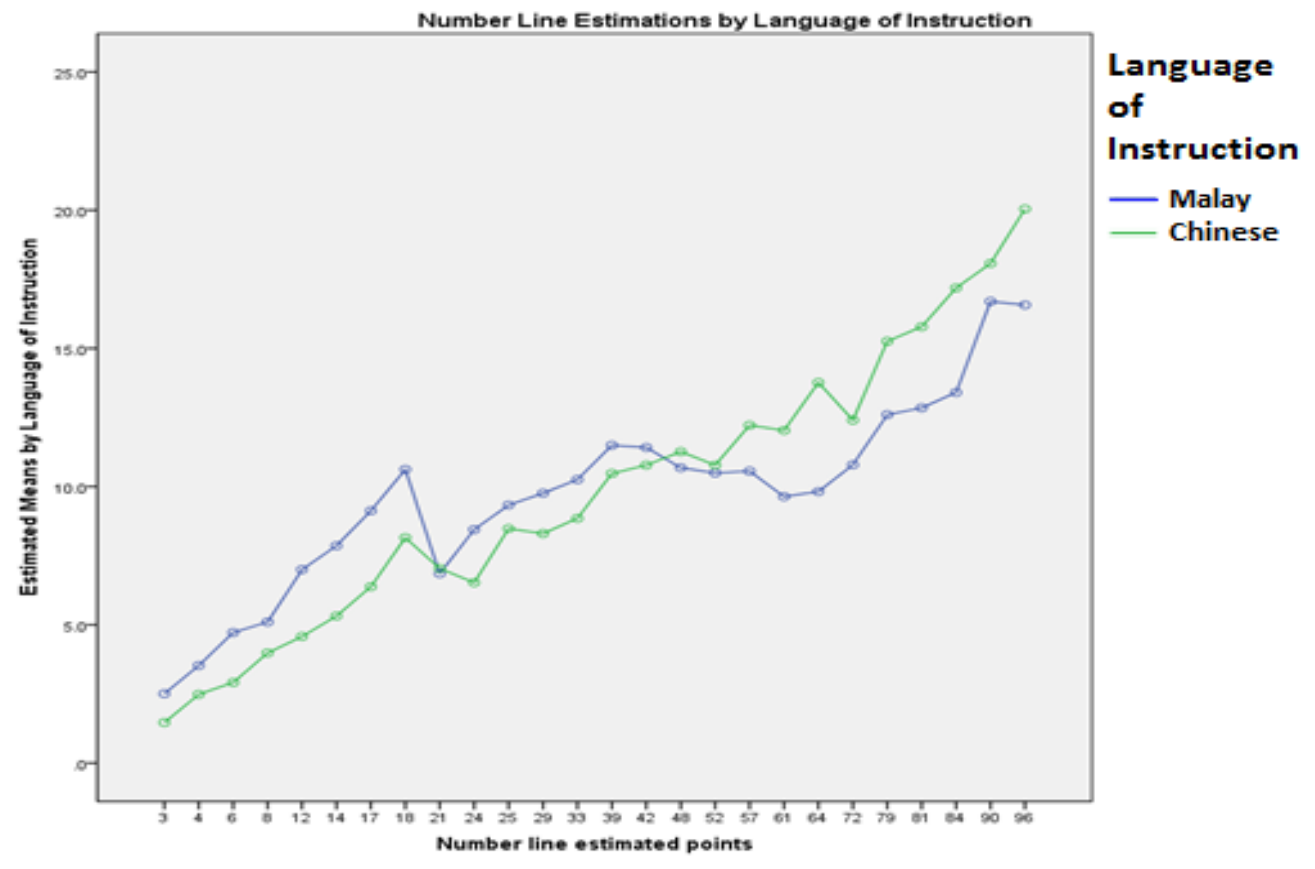

Figure 2. Means of number line estimates by LOI. 


\section{Discussion and Conclusion}

The purpose of this research was to analyze the impact of two different counting systems, Chinese and Malay, on children's learning of the counting string and their understanding of numbers. Both systems are more regular than English and differ in regularity to a smaller degree than counting systems analyzed in previous studies. In spite of the fact that the children lived in the same country and in multilingual environments, the small difference in regularity between the counting systems had an impact on the children's performance.

\section{Counting}

It is worth noting that children in Grade 1 are taught numbers to 100 in school, but the Chinese LOI children performed better on a task that required them to count on from 1000, as well as on the other counting tasks. The majority of the children were able to count to 70 , even though they had just started school. This is in line with past research (Miller et al., 2005), who reported that Chinese 4 year olds could count up to 100. Interestingly, Malay LOI children made more skip errors when counting in the decades. A new word (puluh) is introduced in the decades and the reading of Arabic numerals goes left to right for decades whereas the teens were right to left. This makes the task more taxing on the working memory (Campbell \& Epp, 2004) and thus influences the generation of number names while counting.

In the hundreds board, Malay LOI children tended to not pick up on the clues provided by the numbers in the surrounding boxes both vertically and horizontally. Their mistakes showed that they counted in ones when going across rows or columns, although this is only appropriate for rows. A noteworthy mistake some of the Malay-speaking children made while doing this task was reading the decade numbers from right to left, as if they were teen numbers (e.g., reading 82 as 28). Dowker and colleagues (2008) found similar results and concluded that number systems which require reading Arabic numbers in two different ways from teens to decades interfere with task performance.

\section{Additive Composition}

For the Shop Task, the base-10 system made it easier to combine tens with ones, for it is easier to think about 19 as $10+9$; both number systems provide clues to this combination. However, it is noteworthy that all of the Malay LOI children who made the fives and ones error also made tens and ones error. This suggests an incomplete understanding of their numerical system. Although the Malay LOI children knew the values of the coins and which amount would allow them to buy more things, the idea of additive composition was not entirely achieved at the time. These children also tended to count with ones first rather than count on from five or ten, so Nunes and Bryant's (1996) were also 
observed with this study. Children who used a counting on strategy performed better on additive composition tasks. The results of this research are similar to Miura and collaborators' (1994) and Wang and colleagues' (2008) findings of Chinese children being more likely to use base-10 to construct numbers. They concluded that Chinese children were better at number representation due to the regularities in the numerical naming structure, and this remains true even in a different cultural setting (China Chinese and Malaysian Chinese).

\section{Number Placement}

In terms of predicting numbers on a number line, Chinese LOI children once again made better estimations than their Malay LOI peers. The former tended to estimate larger numbers more accurately than the latter, which shows that Chinese LOI students understood the magnitude of larger numbers. Interestingly, children of both LOI tended to overestimate teen numbers and underestimate twenties, but Malay LOI children underestimated twenties more than Chinese LOI children. This is probably due to a change in both number naming schemes during the decades, for Chinese adds on another syllable (e.g., 21 is 2-10-1), and Malay introduces a new word and switches back to reading numbers from left to right. Similar to what Siegler and Booth (2004) and Siegler and $\mathrm{Mu}$ (2008) found, Chinese children's estimates fit a linear curve and they were better at placing number estimates. A transparent base-10 counting system assists in earlier understanding the size of larger numbers even if across different educational contexts, and this shows that though the Malay numerical scheme is not as regular as Chinese numerical systems, the Malay LOI children's estimates also fit a linear curve as facilitated by the transparency of the base-10 Malay number system.

The results of this study have implications for children in schools of different LOI. Children who use a less regular system (like English) may need to work on additive composition tasks and with the hundreds board in order to attain a better understanding of the system as they learn to count. The use of a counting system that is more regular may lead to this understanding naturally, without the need for much systematic teaching.

\section{References}

Bender, A., \& Beller, S. (2011). Cultural variation in numeration systems and their mapping onto the mental number line. Journal of Cross-Cultural Psychology, 42(4), 579-597. doi:10.1177/0022022111406631

Bryman, A. (2012). Social Research Methods. Oxford: Oxford University Press.

Campbell, J. I. D., \& Epp, L. J. (2004). An encoding-complex approach to numerical cognition in chinese-english bilinguals. Canadian Journal of Experimental Psychology, 58(4), 229-244. doi:10.1037/h0087447 
Cobb, P., Wood, T., Yackel, E., \& McNeal, B. (1992). Characteristics of classroom mathematics traditions: An interactional analysis. American Educational Research Journal, 29, 573-604.

Dowker, A., Bala, S., \& Lloyd, D. (2008). Linguistic influences on mathematical development: how important is the transparency of the counting system? Philosophical Psychology, 21(4), 523-538. doi:10.1080/09515080802285511

Dickson, M., \& Robinson, H. (2013). Early, late or never? When does parental education impact child outcomes ? IZA discussion paper, (7123), 1-47.

Field, A. (2013). Discovering Statistics Using IBM SPSS Statistics (4th editio., pp. 1-870). London, UK: SAGE Publications.

Laski, E. V., \& Siegler, R. S. (2007). Is 27 a big number? Correlational and causal connections among numerical categorization, number line estimation, and numerical magnitude comparison. Child Development, 78, $1723-1743$.

Laski, E. V, \& Yu, Q. (2014). Number line estimation and mental addition: Examining the potential roles of language and education. Journal of experimental child psychology, 117, 29-44. doi:10.1016/j.jecp.2013

Miller, K. F., \& Stigler, J. W. (1987). Counting in chinese: Cultural variation in basic cognitive skill. Cognitive Development, 2, 279-305.

Miller, K. F., Kelly, M., \& Zhou, X. (2005). Learning mathematics in China and the United States: Cross cultural insights into the nature and course of preschool mathematical development. In J. I. . Campbell (Ed.), Handbook of mathematical cognition (pp. 163-178). New York: Psychology Press.

Miura, I. T., Okamoto, Y., Kim, C. C., Chang, C.-M., Steere, M., \& Fayol, M. (1994). Comparisons of children's cognitive representation of number: China, France, Japan, Korea, Sweden, and the United States. International Journal of Behavioral Development, 17(3), 401-411. doi: $10.1177 / 01650254940170$

Nunes, T., \& Bryant, P. (1996). Children doing mathematics. UK: WileyBlackwell.

Pallant, J. (2013). SPSS Survival Manual (5th ed., pp. 1-354). New York, NY: McGraw-Hill.

Seron, X., \& Fayol, M. (1994). Number transcoding in children: A functional analysis. British Journal of Developmental Psychology, 12(3), 281 - 300. doi:10.1111/j.2044-835X.1994.tb00635.x

Siegler, R. S., \& Booth, J. L. (2004). Development of numerical estimation in young children. Child development, 75(2), 428-44. doi:10.1111/j.14678624.2004.00684.X

Siegler, R. S., \& Mu, Y. (2008). Chinese children excel on novel mathematics problems even before elementary school. Psychological Science, 19(8), 759-763. doi:10.1111/j.1467-9280.2008.02153.x

Sui, S. N. N. (2012). The Chinese number naming system and its impact on the arithmetic performance of pre-schoolers in Hong Kong. Mathematics 
Education Research Journal, 24(2), 189-213. doi:10.1007/s13394-0120037-4

Wang, J., Lin, E., Tanase, M., \& Sas, M. (2008). Revisiting the influence of numerical language characteristics on mathematics achievement: Comparison among China, Romania, and U.S. International Electronic Journal of Mathematics Education, 3(1), 24-46.

Zhao, N., Valcke, M., Desoete, A., Burny, E., \& Imbo, I. (2014). Differences between Flemish and Chinese primary students' mastery of basic arithmetic operations. Educational Psychology, 34(7), 1-20.

\section{Appendix A}

Count to 70 task (printed horizontally for actual task with full picture)

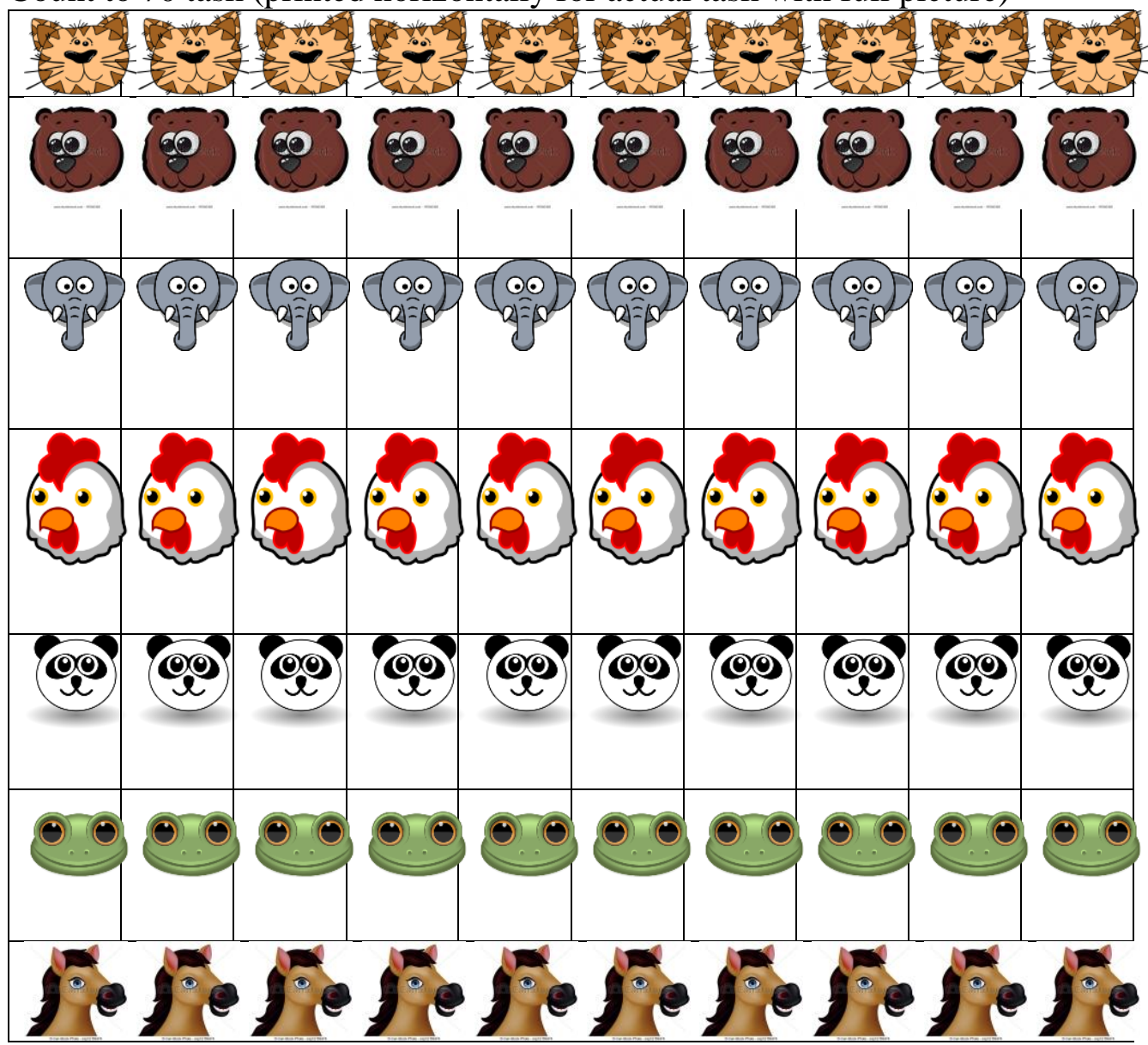




\section{Appendix B}

Hundred Squares Task

\begin{tabular}{|r|r|r|r|r|r|r|r|r|r|}
\hline 0 & 1 & 2 & 3 & 4 & 5 & 6 & 7 & 8 & 9 \\
\hline$*$ & $*$ & 12 & 13 & 14 & $*$ & $*$ & 17 & 18 & 19 \\
\hline 20 & $*$ & 22 & 23 & 24 & 25 & $*$ & $*$ & 28 & 29 \\
\hline 30 & $*$ & 32 & 33 & 34 & 35 & 36 & $*$ & 38 & 39 \\
\hline 40 & 41 & 42 & 43 & 44 & 45 & 46 & 47 & 48 & 49 \\
\hline 50 & 51 & 52 & 53 & 54 & 55 & 56 & 57 & 58 & 59 \\
\hline 60 & 61 & 62 & 63 & 64 & 65 & 66 & 67 & 68 & 69 \\
\hline 70 & 71 & $*$ & 73 & 74 & 75 & 76 & 77 & 78 & 79 \\
\hline 80 & 81 & $*$ & 83 & 84 & 85 & 86 & 87 & 88 & 89 \\
\hline 90 & 91 & 92 & 93 & $*$ & $*$ & 96 & 97 & 98 & 99 \\
\hline
\end{tabular}


Shop Task

\section{Appendix C}

Language of instructions depended on LOI: You'll use the money listed below to buy some things, you will have RM 1, RM 5, RM 10. Please say the exact amount of money you will use.

(a) With fifteen RM1 only.

\section{RM12}

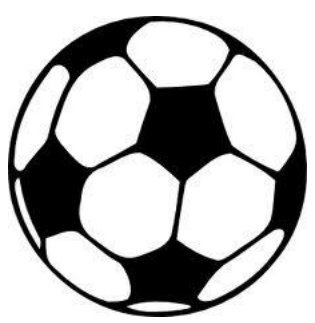

Pay with

(b) With five RM 5 and ten RM1.

\begin{tabular}{|l|l|}
\hline Pay with & Pay with \\
\hline Pay with
\end{tabular}

(c) With three RM10 and ten RM1

\begin{tabular}{|l|l|l|}
\hline \multicolumn{1}{|c|}{ RM14 } & RM19 \\
\hline Pay with & Pay with & Pay with \\
\hline
\end{tabular}


Money used in the shop task.

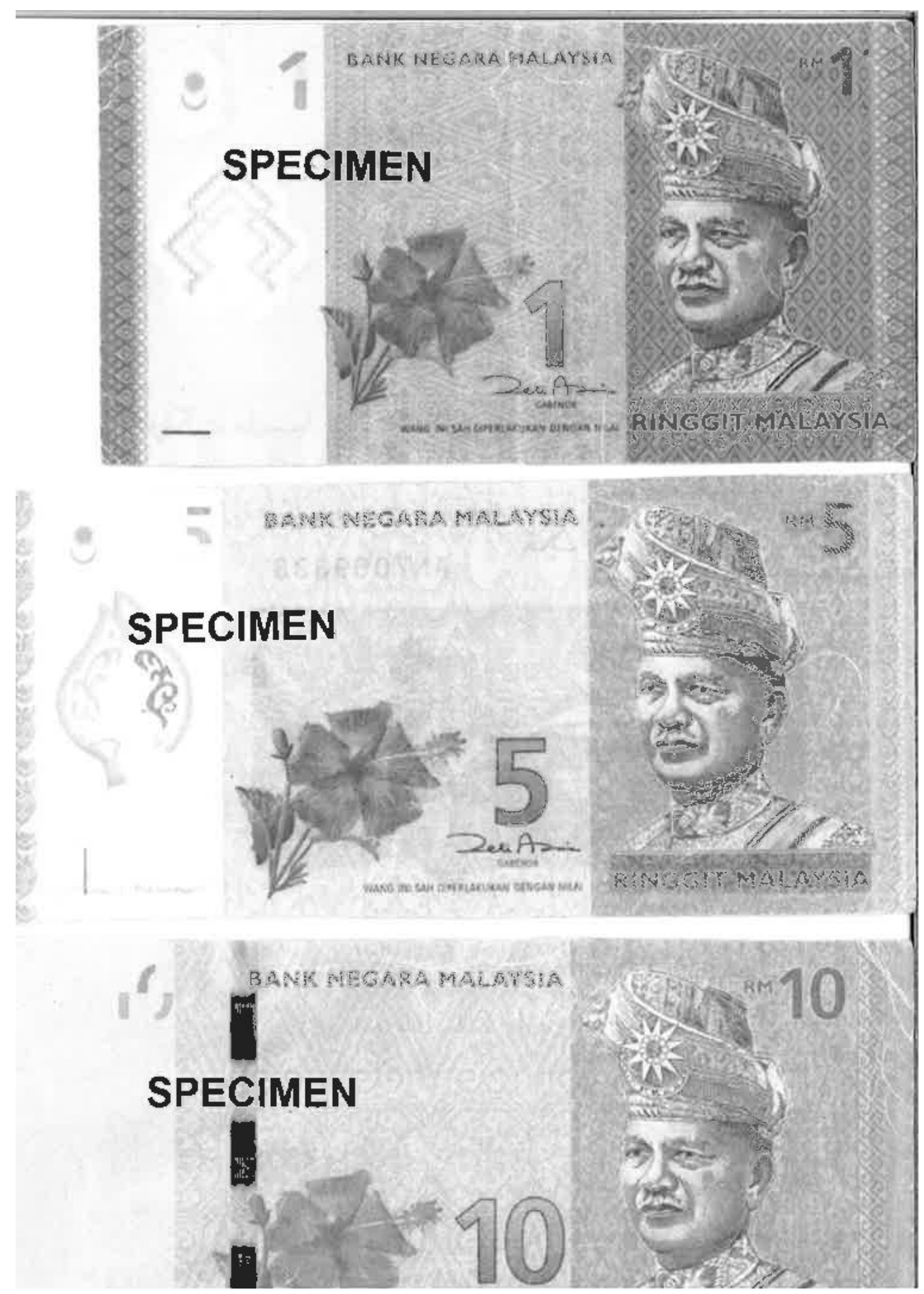




\section{Appendix D}

Number line task (printed horizontally and with more space between questions for actual task)

Instructions: Mark with a line where the following number should be.

(a) 18

12100

(b) 84

1100

(c) 39

1100

(d) 42

1100

(e) 14

12100

(f) 33

$1-100$

(g) 96

$\begin{array}{ll}1 & 100\end{array}$

(h) 90

1100

(i) 17

\begin{tabular}{ll}
\hline 1 & 100
\end{tabular}

(j) 25

1

100

(and so on)

\section{Authors:}

Natalie M. Y. Kheu

University of Oxford

Email: nataliekheu@yahoo.co.uk

Terezinha Nunes

University of Oxford

Email: terezinha.nunes@education.ox.ac.uk 